\title{
Laparoscopic Versus Robotic Roux-En-Y Gastric Bypass: Lessons and Long-Term Follow-Up Learned From a Large Prospective Monocentric Study
}

\author{
Nicolas C. Buchs • Philippe Morel • Dan E. Azagury • \\ Minoa Jung • Gilles Chassot • Olivier Huber • \\ Monika E. Hagen • François Pugin
}

Published online: 26 June 2014

(C) Springer Science+Business Media New York 2014

\begin{abstract}
Background Laparoscopic Roux-en-Y gastric bypass (RYGB) has become the procedure of choice for the treatment of morbid obesity. Recently, several reports have shown the potential advantages of the robotic approach, notably by reducing complications. The aim of this study is to report our long-term experience with robotic Roux-en-Y gastric bypass (RYGB) and to compare outcomes with the laparoscopic approach.

Methods From January 2003 to September 2013, 777 consecutive minimally invasive RYGB have been performed in our institution: 389 laparoscopically (50.1\%) and 388 robotically (49.9\%). During the study period, all the data regarding these consecutive RYGB has been prospectively collected in a dedicated database.

Results While longer in duration compared to laparoscopy (+ $30 \mathrm{~min} ; p=0.0001$ ), the robotic approach had a lower conversion rate $(0.8$ vs. $4.9 \% ; p=0.0007)$, and less complications ( $11.6 \%$ vs. $16.7 \%$; $p=0.05)$, in particular, less gastrointestinal leaks $(0.3$ vs. $3.6 \% ; p=0.0009)$. There were also less early reoperations ( 1 vs. $3.3 \%$; $p=0.05$ ) and a shorter hospital stay in the robotic group ( 6.2 vs. 10.4 days; $p=0.0001)$. There were no statistical differences between the early and the current robotic experience, except in operative time and hospital stay, which were shorter for the last 100 cases. Finally, the BMI loss was significantly higher in the laparoscopic group starting at the first post-operative year.
\end{abstract}

N. C. Buchs $(\bowtie) \cdot$ P. Morel $•$ D. E. Azagury $\cdot$ M. Jung $\cdot$

G. Chassot • O. Huber $\cdot$ M. E. Hagen · F. Pugin

Clinic for Visceral and Transplantation Surgery,

Department of Surgery, University Hospital of Geneva,

Rue Gabrielle-Perret-Gentil 4, 1211 Geneva 14, Switzerland

e-mail: nicolas.c.buchs@hcuge.ch
Conclusions Robotic RYGB is not only safe and feasible, but also a valid option in comparison to laparoscopy. At the cost of a longer operative time, we observed better short-term outcomes with the robotic approach.

Keywords Robot · Laparoscopy · Gastric bypass · Long-term follow up $\cdot$ Weight loss

\section{Introduction}

Twenty years after the first laparoscopic Roux-en-Y gastric bypass (RYGB) [1], it has become the gold standard bariatric intervention $[2,3]$, at least in a majority of bariatric centers. Large series have reported not only good perioperative outcomes, but also very adequate long-term and sustainable weight loss $[4,5]$. In addition, RYGB has been shown to resolve a majority of obesity-related comorbidities such as diabetes, hypertension, and obstructive sleep apnea syndrome $[6,7]$.

Even if the complication rate following laparoscopic RYGB is relatively low, the risk of developing a gastrointestinal leak is still reported up to $5.2 \%[8,9]$. A leak remains as the most dreadful complication after a RYGB, explaining why potential risk factors are still under investigation in order to minimize this risk.

In parallel, robotics has been developed to expand the indications of minimally invasive surgery. Since the early 2000s, robotic surgery has been reported feasible and safe, even for advanced and complex procedures [10]. In bariatric surgery, the robotic technology has been introduced successfully, as reported by several centers [11-15]. Specifically for RYGB, the experience is also positive with a possible reduction of anastomotic 
complications, as confirmed in recent systematic reviews [16-18]. However, the interest remains relatively limited to a few centers, possibly due to the lack of large comparative studies.

The aim of our study was to report our long-term experience with robotic RYGB in a teaching institution and to compare the outcomes with the laparoscopic approach.

\section{Material and Methods}

From January 2003 to September 2013, 777 consecutive minimally invasive RYGB have been performed in our institution: 389 with a laparoscopic approach (50.1\%) and 388 using a robotic approach (49.9\%). During the study period, all the data from the consecutive RYGB has been prospectively collected in a dedicated database.

Of note, we acquired the standard robotic system in 2006 (da Vinci Surgical system, Intuitive Surgical, Sunnyvale, CA) and started robotic RYGB in July 2006 (Fig. 1). Later, the robotic system was upgraded to a da Vinci S system, and finally in 2010 to the da Vinci Si system.

All the procedures were performed by different experienced laparoscopic and robotic surgeons (>100 advanced cases of minimally invasive/bariatric surgery each). Patients included in the bariatric program met the criteria of the Swiss Society for the Study of Morbid Obesity and Metabolic Disorders [19]. All patients underwent a routine preoperative endoscopy as well as a multidisciplinary evaluation including a psychiatric assessment. There were no specific selection criteria for robotics. The exclusion criteria were the same for both groups (anesthesiological contraindication, evident hostile abdomen). There was no randomization. The choice of the approach was based on the availability of the system.

The follow-up was organized by a research nurse especially dedicated to the bariatric program according to our national guidelines.

\section{Surgical Technique}

The surgical technique was standardized and similar between the groups.

The pneumoperitoneum was created using an OPTIVIEW (Endopath Xcel, Ethicon) technique. All patients underwent a routine cholecystectomy.

A small gastric pouch (around $20-30 \mathrm{~cm}^{3}$ ) was created using blue or green cartridge staplers. A standard RYGB with a $150-\mathrm{cm}$ alimentary limb was constructed after the creation of the gastric pouch. In case of robotic approach, a hand-sewn gastrojejunal (GJ) and jejunojejunal (JJ) anastomosis was performed, using a single layer running suture of 2.0 Vicryl (Ethicon). The technique was already described in details elsewhere [12, 20].

For the laparoscopic cases, a mechanical circular anastomosis was preferred with a transorally inserted anvil. Recently, we started to perform linear GJ anastomosis as well. The jejunojejunal anastomosis was performed with a linear stapler.

A routine air leak test was performed at the end of the procedure. A drain was left close to the GJ anastomosis depending on the surgeon's preference.

\section{Postoperative Management}

At the beginning of our experience, patients underwent a routine postoperative liquid contrast swallow at postoperative day (POD) 2. If considered normal, a liquid diet was initiated on POD 3 and puree diet on POD 4. More recently, we decided not to perform an upper gastrointestinal series and to initiate a liquid diet on POD 1, according to our published decisional algorithm [21]. If liquid diet was tolerated on POD 1, patients were advanced to puree diet on POD 2.

They were followed postoperatively at our outpatient clinic at $1,3,6,12$, and 18 months, and annually thereafter.
Fig. 1 Evolution of minimally invasive RYGB through September 2013

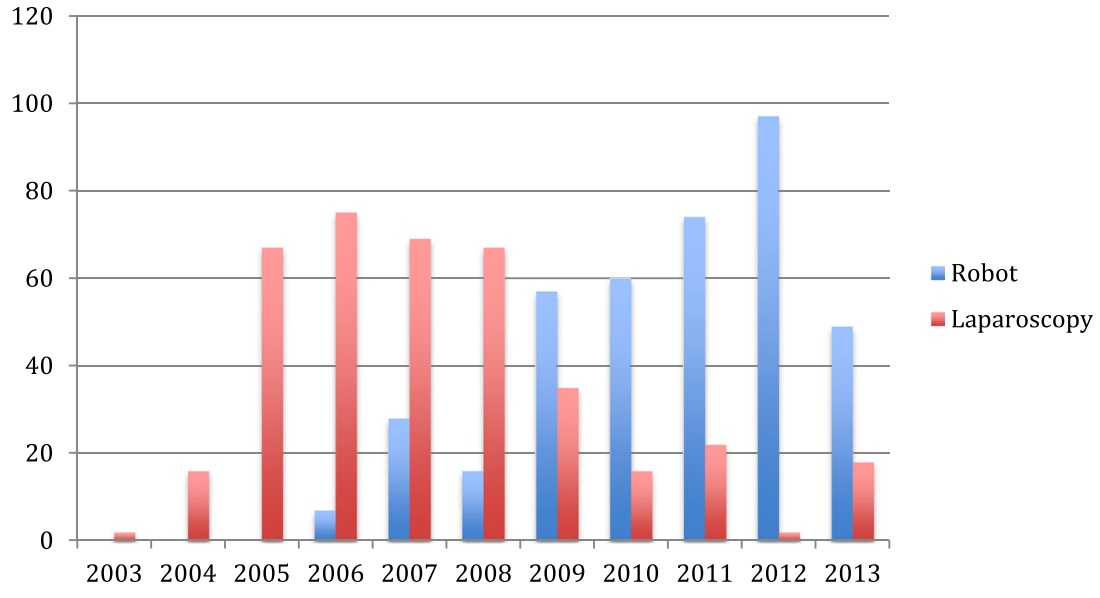




\section{Data Studied}

We evaluated the peri-operative outcomes between both groups. The operative time was defined as the time between the first skin incision and the last skin closure. A conversion was defined as the need to finish the procedure by another approach than the initial one.

We evaluated the 30-day mortality and morbidity, using the Clavien-Dindo classification to grade complications [22].

Regarding weight loss, the percent of BMI loss (\%BMI loss) was defined as the operative BMI minus the follow-up BMI, divided by the operative BMI.

The percent of excess BMI loss (\%EBMIL) was defined as: $100-(($ follow-up BMI-25)/(beginning BMI -25$) \times 100)$, where 25 is defined as the limit of a normal BMI [23].

\section{Statistical Analysis}

The results of parametric and nonparametric data were expressed as mean \pm standard deviation (SD) and median (range), respectively. GraphPad Software (GraphPad, La Jolla, CA) was used for all statistical analyses. Confidence intervals were set at $95 \%$. A two-sided $p$ value of $\leq 0.05$ was considered as statistically significant. Comparisons between both groups were determined using Fisher's exact test for discrete variables and Student's $t$ test for continuous variables.

\section{Results}

During the study period, 777 patients underwent a minimally invasive RYGB, 389 by laparoscopy (50.1\%) and 388 by a robotic approach (49.9\%). Patients' demographics are summarized in Table 1.

There were more male patients in the robotic group, but the difference did not reach statistical significance. On the other hand, patients in the laparoscopic group were slightly younger (42 vs. 43.8 years old for robotics; $p=0.02$ ) and had a higher preoperative BMI $\left(+0.8 \mathrm{~kg} / \mathrm{m}^{2} ; p=0.05\right)$. There were no differences in terms of American Society of Anesthesiologists (ASA) score or comorbidities.

\section{Peri-Operative Outcomes}

The operative time for the robotic approach was longer than laparoscopy (+30 min; $p=0.0001)$ (Table 2). On the other hand, there were less conversions in the robotic group in comparison with laparoscopy ( 0.8 vs. $4.9 \% ; p=0.0007)$. We observed three conversions in the robotic group: one because of a stapler misfire, one because of a non-reducible large hiatal hernia, and finally, one because of severe intra-abdominal adhesions. In the laparoscopic group, 19 conversions were observed: 4 due to severe adhesions, 4 because of a very large left liver lobe, 2 because of difficulties in maintaining an adequate pneumoperitoneum, 1 because of a duodenal injury during the insertion of the initial trocar, and 8 for other various technical problems (for example, stapler misfire).

The intra-operative complication rate was similar in both groups. We had three intra-operative complications in the robotic group. These were a stapler misfiring as mentioned before and an intraoperative perforation of the gastric pouch by the nasogastric tube requiring a robotic redo of the pouch. Finally, one patient presented an intraoperative bronchospasm.

In addition, we observed six intra-operative complications in the laparoscopic group. One patient presented an esophageal lesion during the dissection that was immediately identified and repaired. One patient presented a massive bronchospasm during intubation. One leak was found during the air leak test. One patient presented a duodenal injury during the introduction of the first trocar. One stapler misfire required a conversion to open surgery. And finally, one patient had a paravenous perfusion causing an upper limb compartment syndrome, requiring a fasciotomy.

There was one perioperative death (grade V) in each group $(p=1)$. In the robotic group, a 40-year-old lady was classified ASA 3 and had a BMI of 44. Her past medical history was significant for a coagulopathy, and unfortunately, at postoperative day 1 , she suffered a massive pulmonary embolism and a complete bilateral carotid thrombus. She was immediately treated by endovascular thromboaspiration. Unfortunately, she developed a massive reperfusion cerebral edema and passed away at postoperative day 2. In the laparoscopic group, a 45-year-old lady (ASA 3, BMI 57) developed an early postoperative respiratory distress, caused by an undiagnosed obstructive sleep apnea syndrome. Despite mechanical ventilation and intensive care, a cerebral anoxia developed and she passed away on postoperative day 3 .

Regarding postoperative morbidity, there were less complications in the robotic group (11.6\%) in comparison with laparoscopy $(16.7 \% ; p=0.05)$ (Tables 2 and 3 ).

In the robotic group, we observed 45 complications, with a majority of grades I and II (73.3 \%). We observed 11 grade I complications: anastomotic edemas $(n=3$; requiring a delay in postoperative diet), peripheral paresthesias ( $n=3$; successfully treated conservatively), atelectasis $(n=2)$, and wound problems ( $n=3$; hematoma, abscess, and delayed wound healing). Regarding grade II complications, we observed: pulmonary embolism $(n=12)$ and deep venous thrombosis $(n=2)$, bacteremia ( $n=3$; requiring intravenous antibiotics), urinary tract infections ( $n=2$; requiring antibiotics), peripheral neuropathy $(n=1)$, hematemesis ( $n=1$; necessitating transfusion), and deep parietal abscess ( $n=1$; requiring oral antibiotics). In addition, we observed five grade IIIb complications: four of them required a reoperation as mentioned later. In addition, 
Table 1 Patients' demographics

\begin{tabular}{|c|c|c|c|}
\hline & Robotic $(n=388)$ & Laparoscopy $(n=389)$ & $p$ value \\
\hline Gender & & & 0.09 \\
\hline Male & 104 & 84 & \\
\hline Female & 284 & 305 & \\
\hline Age in years, mean $\pm \mathrm{SD}$ & $43.8 \pm 10.7$ & $42 \pm 10.4$ & 0.02 \\
\hline $\mathrm{BMI}$ in $\mathrm{kg} / \mathrm{m}^{2}$, mean $\pm \mathrm{SD}$ & $44 \pm 5.2$ & $44.8 \pm 6.2$ & 0.05 \\
\hline Weight in $\mathrm{kg}$, mean $\pm \mathrm{SD}$ & $122.1 \pm 20.7$ & $122.8 \pm 21.5$ & 0.64 \\
\hline ASA score, mean \pm SD & $2.3 \pm 0.5$ & $2.3 \pm 0.5$ & 1 \\
\hline \multicolumn{4}{|l|}{ Comorbidity } \\
\hline Diabetes & $81(20.9 \%)$ & $86(22.1 \%)$ & 0.72 \\
\hline Hypertension & $135(34.8 \%)$ & $131(33.7 \%)$ & 0.76 \\
\hline Sleep apnea syndrome & $106(27.3 \%)$ & $85(21.9 \%)$ & 0.6 \\
\hline
\end{tabular}

$S D$ standard deviation, $B M I$ body mass index, $A S A$ American Society of Anesthesiology

one patient presented a bleeding anastomotic ulcer requiring endoscopic hemostasis. Finally, we observed six grade IVa complications: respiratory failure $(n=3$; requiring a prolonged intubation in the intensive care unit (ICU)), severe pneumonia $(n=1$; necessitating intubation), pulmonary embolism $(n=1$; requiring 5 days of monitoring in the ICU), and laryngeal edema ( $n=1$; necessitating prolonged intubation).

In the laparoscopic group, 65 complications were recorded. The majority were grades I and II (69.2\%). Regarding grade I complications, we observed: wound abscess $(n=8)$, self-limited GJ leak $(n=5$; treated conservatively without removing the drain), atelectasis $(n=2)$, bile leak following a liver biopsy $(n=$ 1 ; treated successfully by keeping the drain in place), phlebitis $(n=1)$, postoperative agitation $(n=1)$, and edema at the level of the GJ ( $n=1$; delaying the postoperative diet). Regarding grade II complications, we observed: pulmonary embolisms $(n=8)$ and deep venous thrombosis $(n=1)$, gastrointestinal bleeding $(n=4$; requiring only blood transfusion and monitoring), urinary tract infection ( $n=4$; necessitating oral antibiotics), pneumonias ( $n=$ 4 ), intra-abdominal abscess ( $n=2$; requiring intravenous antibiotics), heparin-induced thrombocytopenia ( $n=1$; requiring oral anticoagulation), acute pulmonary edema ( $n=1$; necessitating oral diuretics and physiotherapy), and diarrhea with positive culture for Clostridium difficile $(n=1)$. Twelve grade III complications were observed. Three of them were graded IIIa: one duodenal leak after subtotal gastrectomy during the RYGB (resection for multiple polyps) requiring a radiological drainage. One pulmonary embolism necessitated a vena cava filter. And finally, one bile leak (from a liver biopsy) was diagnosed and required radiological drainage. Nine grade IIIb complications were also observed; all of them required a reoperation as discussed later. Seven grade IV complications were reported. Two were graded IVa: one respiratory insufficiency requiring intubation, and one prolonged intubation in the ICU for another patient. Finally, five complications were graded IVb: four of them due to a gastrointestinal leak with septic shock requiring a reoperation as mentioned below. Another patient presented a pulmonary embolism with acute respiratory distress, associated with kidney failure.

Table 2 Perioperative outcomes

\begin{tabular}{llll}
\hline & Robotic $(n=388)$ & Laparoscopy $(n=389)$ & $p$ value \\
\hline Operative time in minutes, mean \pm SD & $245 \pm 93.6$ & $215.8 \pm 69.1$ & 0.0001 \\
Conversion rate & $3(0.8 \%)$ & $19(4.9 \%)$ & 0.0007 \\
Intraoperative complications & $3(0.8 \%)$ & $6(1.5 \%)$ & 0.5 \\
Postoperative complications & $45(11.6 \%)$ & $65(16.7 \%)$ & 0.05 \\
$\quad$ Gastrointestinal leak & $1(0.3 \%)$ & $14(3.6 \%)$ & 0.0009 \\
$\quad$ Pulmonary embolism & $17(4.4 \%)$ & $9(2.3 \%)$ & 0.12 \\
30-day mortality & $1(0.3 \%)$ & $1(0.3 \%)$ & 1 \\
Reoperations & $4(1 \%)$ & $13(3.3 \%)$ & 0.05 \\
Length of stay in days, mean \pm SD & $6.2 \pm 4.8$ & $10.4 \pm 17.5$ & 0.0001 \\
\hline
\end{tabular}

$S D$ standard deviation

Italic numbers were given for statistically significant comparisons 
Table 3 30-Day postoperative complications according to ClavienDindo classification [22]

\begin{tabular}{llll}
\hline Grade & Robotic $(n=45)$ & Laparoscopy $(n=65)$ & $p$ value \\
\hline I & 11 & 19 & 0.66 \\
II & 22 & 26 & 0.4 \\
IIIa & 0 & 3 & 0.27 \\
IIIb & 5 & 9 & 0.77 \\
IVa & 6 & 2 & 0.06 \\
IVb & 0 & 5 & 0.08 \\
V & 1 & 1 & 1 \\
\hline
\end{tabular}

In addition, there were less early reoperations in the robotic groups $(1 \%)$ in comparison with laparoscopy $(3.3 \% ; p=0.05)$. In the robotic group, we observed four reinterventions: one due to a staple line bleed, one because of an incarcerated port site hernia, one because of a late GJ leak (at POD 13 after a large meal), and one for a suspected infected hematoma. In the laparoscopic group, we found 13 reoperations. Eight of them were performed for intestinal leaks (four JJ anastomotic leaks, three GJ anastomotic leaks, and one at the level of the remnant stomach). One patient underwent a reoperation for suspicion of intestinal leak, but with no intra-operative finding. We noticed one reoperation for an incarcerated port site hernia at POD 3, leading to a GJ blowout because of the overpressure. One patient presented a cystic duct leak on POD 1, requiring a reoperation. One patient underwent a reoperation for a large hematoma, necessitating exploratory laparoscopy and drainage. Finally, one patient presented a kinking at the level of the common intestinal limb, resulting in a mechanical ileus requiring a reoperation and remnant gastrostomy.

\section{Effect of the Learning Curve}

We chronologically divided our robotic and laparoscopic cases in groups of 100 patients. When comparing our initial cases with our current experience, we found no differences in terms of complications, reoperation, and mortality rates (Table 4). On the other hand, there was a strong statistical difference in operative time: minus $2 \mathrm{~h}$ for the comparison between the first 100 robotic cases and the last 100 robotic cases $(p=0.0001)$. Similarly, the difference between the last 100 laparoscopic cases and the last 100 robotic cases tends to disappear (only a difference of $18 \mathrm{~min} ; p=0.06$ ). There were fewer conversions in the last robotic cases in comparison with the last laparoscopic cases as well ( 0 vs. $8 \% ; p=0.007)$. Finally, there was a shorter hospital stay for the robotic group in comparison to laparoscopy, whatever the studied period.
Effect on the Body Mass Index

The maximum BMI loss was observed at 24 months (Table 5). It is interesting to note that the laparoscopic group tends to present a higher BMI loss, a higher percent BMI loss, and a higher percent excess BMI loss in comparison to the robotic group.

\section{Discussion}

A decade after the first robotic bariatric procedure, robotics is still looking to find its place in the bariatric surgeon's armamentarium. The feasibility and the safety of the robotic approach have been clearly established not only for RYGB [11-15], but also for gastric banding [24], sleeve gastrectomy [25], and duodenal switch [26]. Despite these encouraging reports, part of the surgical community still awaits stronger evidence of benefits of the robotic technique. Recently, several systematic reviews reported at least similar [18, 27], if not better $[16,17]$, postoperative outcomes thanks to the robotic technology. In addition, other possible advantages were reported: the learning curve could be shortened $[13,18,20,28$, $29]$, the cost could be minimized in some centers [12, 21], and particularly difficult procedures such as for superobese patients [30] and revisional procedures [17, 31, 32] can be performed safely.

In summary, we report herein one of the largest comparative studies, evaluating the robotic and laparoscopic approaches. In addition, this series presents one of the longest follow-ups to date. The lessons learned from this large experience are important, and the adoption of the robotic technology in our center allows us to improve our outcomes. These results tend to be within the range of other comparative series (Table 6). Undoubtedly, the operative time for the robotic approach remains longer than standard laparoscopy, even if recent systematic reviews failed to show any differences [16, 27]. While this difference tends to disappear with time, this phenomenon was almost uniformly reported in other series [12, 28, 33, 36-38]. A longer operative time can be explained, at least in part, by the addition of the docking time and the need to change instruments regularly.

More interestingly, the conversion rate can be significantly reduced (less than $1 \%$ for robotic versus almost $5 \%$ for laparoscopy). Beyond the possible effect of the learning curve, robotics could help to minimize the risk of conversion, as reported for other indications [39]. More interestingly, the complication rate can be reduced thanks to the robotic technology, especially the risk of anastomotic leak. In our series, we observed only one late GJ leak and no JJ leak in the robotic group, in comparison to $13 \mathrm{GJ}$ and JJ leaks in the laparoscopic group. Even if the clinical significance of several leaks in the 
Table 4 Differences between the first 100 cases and the last 100 cases

\begin{tabular}{|c|c|c|c|c|c|}
\hline & Robotic first 100 cases & Robotic last 100 cases & Laparoscopy first 100 cases & Laparoscopy last 100 cases & $p$ value \\
\hline Operative time & $335 \pm 97.3$ & $217 \pm 79.4$ & $269 \pm 80.6$ & $199 \pm 51.9$ & $0.0001^{*}$ \\
\hline Conversion rate & $2(2 \%)$ & 0 & $4(4 \%)$ & $8(8 \%)$ & $\mathrm{NS}^{* *}$ \\
\hline Intra-operative complications & 0 & $1(1 \%)$ & 0 & $3(3 \%)$ & NS \\
\hline Postoperative complications & $15(15 \%)$ & $12(12 \%)$ & $16(16 \%)$ & $16(16 \%)$ & NS \\
\hline Leak & 0 & $1(1 \%)$ & $5(5 \%)$ & $3(3 \%)$ & NS \\
\hline PE & $6(6 \%)$ & $4(4 \%)$ & $2(2 \%)$ & $3(3 \%)$ & NS \\
\hline Mortality & 0 & 0 & 0 & $1(1 \%)$ & NS \\
\hline Reoperation & $1(1 \%)$ & $2(2 \%)$ & $4(4 \%)$ & $5(5 \%)$ & NS \\
\hline Length of stay & $8 \pm 2.7$ & $6 \pm 8.3$ & $13 \pm 20.9$ & $9 \pm 11.6$ & $<0.05^{* * *}$ \\
\hline
\end{tabular}

Expressed in mean \pm standard deviation

$P E$ pulmonary embolism, $N S$ not significant

*Highly statistically significant, except for the comparison between 100 last robotic cases and 100 last laparoscopic cases $(p=0.06)$

**Statistically significant between 100 last robotic cases and 100 last laparoscopic cases $(p=0.007)$

***Statistically significant, except the comparison between 100 first laparoscopic cases and 100 last laparoscopic cases $(p=0.1)$

laparoscopic group was limited (successful conservative treatment), a robotic hand-sewn anastomosis seems safer than a laparoscopic mechanical one. This reduced risk was also reported by other groups $[12,14,28,29]$. To illustrate, Tieu et al. [11] found an anastomotic leak rate of only $0.09 \%$ in the largest robotic series to date. In addition, in a large comparative study, Snyder et al. [14] reported a significant difference in terms of anastomotic leaks. Indeed, they found no leak in the robotic group versus $1.7 \%$ of leakage in the laparoscopic group $(p=0.05)$.
On the other hand, the risk of pulmonary embolism was almost twice as high following robotic approach in comparison with laparoscopy. This could be explained by a longer operative time in the robotic group, as management and prophylaxis was otherwise similar between both groups. Finally, in terms of potential advantages, we observed fewer reoperations (threefold reduction) and a shorter hospital stay (minus 4 days) in the robotic group. These findings are consistent with the current robotic literature (Table 6), even if less evident in other series. Moreover, the effect of the learning

Table 5 Evolution of body mass index

\begin{tabular}{|c|c|c|c|}
\hline & Robotic group & Laparoscopic group & $p$ value \\
\hline Number of patients at 1 month & 343 & 353 & \\
\hline BMI loss at 1 month, mean \pm SD & $4.8 \pm 1.6$ & $5 \pm 1.8$ & 0.12 \\
\hline Percent BMI loss at 1 month (\%), mean \pm SD & $10.9 \pm 3.3$ & $11.1 \pm 3.6$ & 0.44 \\
\hline Percent excess BMI loss at 1 month (\%), mean \pm SD & $26.2 \pm 9$ & $26.3 \pm 9.2$ & 0.89 \\
\hline Number of patients at 12 months & 257 & 280 & \\
\hline BMI loss at 12 months, mean \pm SD & $14.7 \pm 4.2$ & $15.9 \pm 3.9$ & 0.0007 \\
\hline Percent BMI loss at 12 months $(\%)$, mean \pm SD & $33.3 \pm 8.5$ & $35.5 \pm 7$ & 0.001 \\
\hline Percent excess BMI loss at 12 months (\%), mean \pm SD & $79.7 \pm 23.1$ & $83.9 \pm 19$ & 0.02 \\
\hline Number of patients at 24 months & 140 & 219 & \\
\hline BMI loss at 24 months, mean \pm SD & $15.3 \pm 4.4$ & $17.1 \pm 5.5$ & 0.001 \\
\hline Percent BMI loss at 24 months ( $\%)$, mean \pm SD & $34.7 \pm 8.2$ & $37.8 \pm 10.1$ & 0.002 \\
\hline Percent excess BMI loss at 24 months (\%), mean \pm SD & $83.5 \pm 21.1$ & $87.4 \pm 25$ & 0.12 \\
\hline Number of patients at 36 months & 75 & 175 & \\
\hline BMI loss at 36 months, mean \pm SD & $13.8 \pm 4.2$ & $16.1 \pm 4.8$ & 0.0004 \\
\hline Percent BMI loss at 36 months $(\%)$, mean \pm SD & $31.5 \pm 8.7$ & $35.8 \pm 8.3$ & 0.0003 \\
\hline Percent Excess BMI loss at 36 months (\%), mean \pm SD & $75.2 \pm 22$ & $83.6 \pm 19.5$ & 0.003 \\
\hline
\end{tabular}

$B M I$ body mass index, $S D$ standard deviation

Italic numbers were given for statistically significant comparisons 
Table 6 Comparative series ( $>10$ patients) evaluating robotic and laparoscopic RYGB

\begin{tabular}{|c|c|c|c|c|c|c|c|}
\hline Authors & Approach & Number & ORT & Conversion $(\%)$ & Complications (\%) & Leaks (\%) & LOS \\
\hline \multirow{2}{*}{ Artuso et al. [33] } & $\mathrm{R}$ & 41 & 289 & NA & NA & $<3$ & NA \\
\hline & $\mathrm{L}$ & 120 & 174 & NA & NA & $<3$ & NA \\
\hline \multirow[t]{2}{*}{ Ayloo et al. [13] } & $\mathrm{R}$ & 90 & 207 & 0 & 2.2 & 0 & 2 \\
\hline & $\mathrm{L}$ & 45 & 227 & 0 & $11 *$ & 0 & 3 \\
\hline \multirow[t]{2}{*}{ Benizri et al. [34] } & $\mathrm{R}$ & 100 & 130 & 3 & 24 & 3 & 9.3 \\
\hline & $\mathrm{L}$ & 100 & 147 & 1 & 21 & 0 & 6.7 \\
\hline \multirow[t]{3}{*}{ Curet et al. [35] } & $\mathrm{R}$ & 21 & 181.7 & NA & 14.3 & NA & 3 \\
\hline & L (sutured) & 36 & 183.3 & NA & 16.7 & NA & 3.3 \\
\hline & L (stapled) & 78 & 185.6 & NA & 19.2 & NA & 3.6 \\
\hline \multirow[t]{2}{*}{ Hagen et al. [12] } & $\mathrm{R}$ & 143 & 293 & 1.4 & 16.1 & 0 & 7.4 \\
\hline & $\mathrm{L}$ & 323 & 206 & 4.9 & 18 & 4 & 11 \\
\hline \multirow[t]{2}{*}{ Hubens et al.[28] } & $\mathrm{R}$ & 45 & 212 & 20 & 6.7 & 0 & 4.7 \\
\hline & $\mathrm{L}$ & 45 & 127 & 0 & 13.3 & 4.4 & 4.7 \\
\hline \multirow[t]{2}{*}{ Myers et al. [36] } & $\mathrm{R}$ & 100 & 144 & 0 & NA & 1 & 1.5 \\
\hline & $\mathrm{L}$ & 100 & 87 & 0 & NA & 1 & 2.2 \\
\hline \multirow[t]{2}{*}{ Mohr et al. [29] } & $\mathrm{R}$ & 10 & 169 & 40 & 20 & 0 & NA \\
\hline & $\mathrm{L}$ & 10 & 208 & 50 & 20 & 10 & NA \\
\hline \multirow[t]{2}{*}{ Park et al. [37] } & $\mathrm{R}$ & 105 & 169 & 1 & 9.5 & 1.9 & 3.4 \\
\hline & $\mathrm{L}$ & 195 & 152 & 1.5 & 9.7 & 2.1 & 3 \\
\hline \multirow[t]{2}{*}{ Sanchez et al. [15] } & $\mathrm{R}$ & 25 & 130.8 & 0 & 0 & 0 & 2.9 \\
\hline & $\mathrm{L}$ & 25 & 149.4 & 0 & 0 & 0 & 2.6 \\
\hline \multirow[t]{2}{*}{ Scozzari et al. [38] } & $\mathrm{R}$ & 110 & 247.5 & 0 & 16.4 & 1.8 & 7.8 \\
\hline & $\mathrm{L}$ & 423 & 187 & NA & NA & 1.9 & 8.3 \\
\hline \multirow[t]{2}{*}{ Snyder et al. [14] } & $\mathrm{R}$ & 320 & 192 & NA & 22.5 & 0 & 2.7 \\
\hline & $\mathrm{L}$ & 356 & NA & NA & 21.6 & 1.7 & 3 \\
\hline
\end{tabular}

$R$ robotic, $L$ laparoscopic, $O R T$ operative time in minutes, $L O S$ length of stay in days, $N A$ not available

*Including late complications

curve was low, since only the operative time and the length of stay were different between the early and the most recent experience.

A new element remains: the difference between both groups in terms of BMI loss. Indeed, the laparoscopic group presented a higher BMI loss. While this was a surprise for our group, several reasons might be hypothesized. Even if the technique was similar between both approaches, a couple of differences exist: first, the anastomosis was hand-sewn with absorbable sutures in the robotic group and could be less obstructive than a circular mechanical anastomosis, especially in the long term. Indeed, the effect of the stoma diameter is known to be a potential risk factor for weight regain [40]. Thus, we are evaluating a calibration of the GJ anastomosis in order to avoid too-large stoma. In addition, the length of the alimentary limb might be shorter in the robotic group. Indeed, the enlarged three-dimensional vision could render the measurement of the alimentary limb more difficult than in the standard laparoscopic group. In order to try and correct this hypothetical parameter, we have now started measuring the length of the different segments with an umbilical tape or a marked instrument. On the other hand, Park et al. [37] did not find any significant differences in terms of weight loss at 1 year after robotic RYGB.

Clearly, in 2013, the real question is to determine the best indications for the robotic technology. The present series gives encouraging results, showing positive shortterm outcomes with diminished leak rate. Despite these promising results, robotics still has some difficulties in establishing itself as the gold standard approach. Several reasons have been proposed to tentatively explain this fact. First, skeptical readers might argue that only few evidences and controversial results are available to date. In fact, several comparative studies (Table 6) were published and reported good outcomes after a robotic approach. More interesting, the majority of these series have shown clear advantages of using the robotic technology, as confirmed by systematic reviews [16, 18]. 
With a cumulative experience of more than 1,800 robotic RYGB reported in the literature, the robotic approach can be considered as safe, feasible, and a valid option, especially for difficult cases. Indeed, as mentioned earlier, revisional procedures, which are typically challenging, can be performed safely [31] and with better outcomes in comparison to open or laparoscopy [32]. Super obese patients, with a higher peri-operative risk, could be another good indication, notably by reducing the torque effect and allowing the surgeon to work in a deep and narrow space [30].

On the other hand, criticisms have been raised concerning the excessive costs of the robotic technology [41]. In the bariatric field, we have recently reported our cost analysis that showed a clear advantage of the robotic approach, notably by reducing complications [12] and by minimizing the need of postoperative radiological examinations [21]. Other groups have also found similar total hospital charges [37]. However, these findings were not confirmed by other series that show higher costs [28, 35, 38]. Recently, Bailey et al. [27] performed a systematic review and economic analysis showing that the expected costs for robotic RYGB were higher than that for laparoscopy. More data is clearly required to evaluate the exact economical role of robotics in bariatric surgery.

While bringing new data, this study has several limitations that require comments. First, by its non-randomized nature, this series remains only a large prospective comparative study. Different periods of time and different surgeons were taken into consideration. Yet, we used the same standardized technique, reproducible in a teaching institution. Globally, there were no significant differences between the various periods considered, except for the operative time and the length of stay (effect of the learning curve?).

Then, even if standardized, the technique between both groups was not exactly the same. Indeed, the anastomotic technique was different: mechanical versus hand-sewn. This bias [42] could explain, at least in part, the difference of outcomes. Indeed, Ayloo et al. [13] have reported a handsewn anastomotic technique in both groups, with similar results, except an operative time shorter for the robotic group. We also note that our laparoscopic operative time remains typically longer than previously reported (Table 6).

\section{Conclusions}

We present herein one of the largest robotic series with longterm follow-up. We have shown that robotic RYGB is not only safe and feasible, but also a valid option in comparison to laparoscopy. At the price of a longer operative time, we observed better short-term outcomes after a robotic approach. On the other hand, the long-term outcomes regarding the BMI loss were surprisingly in disfavor of the robotic approach, motivating a modification of our technique (measurement of the alimentary limb, calibration of the GJ anastomosis).

Acknowledgments We would like to thank Pascale Koutny-Fong, research nurse, for the data collection.

Conflict of Interest Drs. Nicolas C. Buchs, Philippe Morel, Dan Azagury, Minoa Jung, Gilles Chassot, Olivier Huber, and François Pugin have no conflict of interest or financial ties to disclose. Monika E. Hagen has a financial relationship with Intuitive Surgical Inc.

\section{References}

1. Wittgrove AC, Clark GW, Tremblay LJ. Laparoscopic gastric bypass, Roux-en-Y: preliminary report of five cases. Obes Surg. 1994;4(4): 353-7.

2. Powell MS, Fernandez AZ. Surgical treatment for morbid obesity: the laparoscopic Roux-en-Y gastric bypass. Surg Clin N Am. 2011;91(6):1203-24.

3. Buchwald H, Williams SE. Bariatric surgery worldwide 2003. Obes Surg. 2004;14(9):1157-64.

4. Schauer P, Conference ABSC. Gastric bypass for severe obesity: approaches and outcomes. Surg Obes Relat Dis. 2005;1(3):297-300.

5. Schauer PR, Ikramuddin S. Laparoscopic surgery for morbid obesity. Surg Clin N Am. 2001;81(5):1145-79.

6. Buchwald H, Estok R, Fahrbach K, et al. Weight and type 2 diabetes after bariatric surgery: systematic review and meta-analysis. Am J Med. 2009;122(3):248-56.

7. Sjostrom L, Lindroos AK, Peltonen M, et al. Lifestyle, diabetes, and cardiovascular risk factors 10 years after bariatric surgery. N Engl J Med. 2004;351(26):2683-93.

8. Lee S, Carmody B, Wolfe L, et al. Effect of location and speed of diagnosis on anastomotic leak outcomes in 3828 gastric bypass cases. J Gastrointest Surg. 2007;11(6):708-13.

9. Jones Jr KB, Afram JD, Benotti PN, et al. Open versus laparoscopic Roux-en-Y gastric bypass: a comparative study of over 25,000 open cases and the major laparoscopic bariatric reported series. Obes Surg. 2006;16(6):721-7.

10. Buchs NC, Addeo P, Bianco FM, et al. Perioperative risk assessment in robotic general surgery: lessons learned from 884 cases at a single institution. Arch Surg. 2012;147(8):701-8.

11. Tieu K, Allison N, Snyder B, et al. Robotic-assisted Roux-en-Y gastric bypass: update from 2 high-volume centers. Surg Obes Relat Dis. 2013;9(2):284-8.

12. Hagen ME, Pugin F, Chassot G, et al. Reducing cost of surgery by avoiding complications: the model of robotic Roux-en-Y gastric bypass. Obes Surg. 2012;22(1):52-61.

13. Ayloo SM, Addeo P, Buchs NC, et al. Robot-assisted versus laparoscopic Roux-en-Y gastric bypass: is there a difference in outcomes? World J Surg. 2011;35(3):637-42.

14. Snyder BE, Wilson T, Leong BY, et al. Robotic-assisted Roux-en-Y Gastric bypass: minimizing morbidity and mortality. Obes Surg. 2010;20(3):265-70.

15. Sanchez BR, Mohr CJ, Morton JM, et al. Comparison of totally robotic laparoscopic Roux-en-Y gastric bypass and traditional laparoscopic Roux-en-Y gastric bypass. Surg Obes Relat Dis. 2005;1(6): 549-54.

16. Markar SR, Karthikesalingam AP, Venkat-Ramen V, et al. Robotic vs. laparoscopic Roux-en-Y gastric bypass in morbidly obese patients: systematic review and pooled analysis. Int J Med Robot. 2011;7(4):393-400. 
17. Kim K, Hagen ME, Buffington C. Robotics in advanced gastrointestinal surgery: the bariatric experience. Cancer J. 2013;19(2):177-82.

18. Fourman MM, Saber AA. Robotic bariatric surgery: a systematic review. Surg Obes Relat Dis. 2012;8(4):483-8.

19. Swiss Study Group for Morbid Obesity (SMOB). Directives pour le traitement chirurgical de l'obésité. www.smob.ch 2010.

20. Buchs NC, Pugin F, Bucher P, et al. Learning curve for robot-assisted Roux-en-Y gastric bypass. Surg Endosc. 2012;26(4):1116-21.

21. Buchs NC, Bucher P, Pugin F, et al. Value of performing routine postoperative liquid contrast swallow studies following robotassisted Roux-en-Y gastric bypass. Swiss Med Wkly. 2012;142: w13556.

22. Dindo D, Demartines N, Clavien PA. Classification of surgical complications: a new proposal with evaluation in a cohort of 6336 patients and results of a survey. Ann Surg. 2004;240(2):205-13.

23. Deitel M, Greenstein RJ. Recommendations for reporting weight loss. Obes Surg. 2003;13(2):159-60.

24. Edelson PK, Dumon KR, Sonnad SS, et al. Robotic vs. conventional laparoscopic gastric banding: a comparison of 407 cases. Surg Endosc. 2011;25(5):1402-8.

25. Ayloo S, Buchs NC, Addeo P, et al. Robot-assisted sleeve gastrectomy for super-morbidly obese patients. J Laparoendosc Adv Surg Tech A. 2011;21(4):295-9.

26. Sudan R, Bennett KM, Jacobs DO, et al. Multifactorial analysis of the learning curve for robot-assisted laparoscopic biliopancreatic diversion with duodenal switch. Ann Surg. 2012;255(5):940-5.

27. Bailey JG, Hayden JA, Davis PJB, et al. Robotic versus laparoscopic Roux-en-Y gastric bypass (RYGB) in obese adults ages 18 to 65 years: a systematic review and economic analysis. Bailey Surg Endosc 2014;28:414-26.

28. Hubens G, Balliu L, Ruppert M, et al. Roux-en-Y gastric bypass procedure performed with the da Vinci robot system: is it worth it? Surg Endosc. 2008;22(7):1690-6.

29. Mohr CJ, Nadzam GS, Curet MJ. Totally robotic Roux-en-Y gastric bypass. Arch Surg. 2005;140(8):779-86.
30. Buchs NC, Pugin F, Chassot G, et al. Robot-assisted Roux-en-Y gastric bypass for super obese patients: a comparative study. Obes Surg. 2013;23(3):353-7.

31. Snyder B, Wilson T, Woodruff V, et al. Robotically assisted revision of bariatric surgeries is safe and effective to achieve further weight loss. World J Surg. 2013;37(11):2569-73.

32. Buchs NC, Pugin F, Azagury DE, et al. Robotic revisional bariatric surgery: a comparative study with laparoscopic and open surgery. Int J Med Robot 2014;10:213-7.

33. Artuso D, Wayne M, Grossi R. Use of robotics during laparoscopic gastric bypass for morbid obesity. JSLS. 2005;9(3):266-8.

34. Benizri EI, Renaud M, Reibel N, et al. Perioperative outcomes after totally robotic gastric bypass: a prospective nonrandomized controlled study. Am J Surg. 2013;206(2):145-51.

35. Curet MJ, Curet M, Solomon H, et al. Comparison of hospital charges between robotic, laparoscopic stapled, and laparoscopic hand-sewn Roux-en-Y gastric bypass. J Robot Surg. 2009;3:75-8.

36. Myers SR, McGuirl J, Wang J. Robot-assisted versus laparoscopic gastric bypass: comparison of short-term outcomes. Obes Surg. 2013;23(4):467-73.

37. Park CW, Lam EC, Walsh TM, et al. Robotic-assisted Roux-en-Y gastric bypass performed in a community hospital setting: the future of bariatric surgery? Surg Endosc. 2011;25(10):3312-21.

38. Scozzari G, Rebecchi F, Millo P, et al. Robot-assisted gastrojejunal anastomosis does not improve the results of the laparoscopic Rouxen-Y gastric bypass. Surg Endosc. 2011;25(2):597-603.

39. Baik SH, Kwon HY, Kim JS, et al. Robotic versus laparoscopic low anterior resection of rectal cancer: short-term outcome of a prospective comparative study. Ann Surg Oncol. 2009;16(6):1480-7.

40. Abu Dayyeh BK, Lautz DB, Thompson CC. Gastrojejunal stoma diameter predicts weight regain after Roux-en-Y gastric bypass. Clin Gastroenterol Hepatol. 2011;9(3):228-33.

41. Barbash GI, Glied SA. New technology and health care costs - the case of robot-assisted surgery. N Engl J Med. 2010;363(8):701-4.

42. Addeo P, Buchs NC. Robotic and laparoscopic gastric bypass: are they comparable? Surg Endosc. 2012;26(2):576-7. 